\title{
Multidamage Detection of Bridges Using Rough Set Theory and Naive-Bayes Classifier
}

\author{
Shuang Sun, Li Liang ${ }^{D}$, Ming Li, and Xin Li \\ School of Resources and Civil Engineering, Northeastern University, Shenyang 110819, China \\ Correspondence should be addressed to Li Liang; ll-neu@163.com
}

Received 10 December 2017; Revised 3 April 2018; Accepted 10 April 2018; Published 27 May 2018

Academic Editor: Filippo Ubertini

Copyright (C) 2018 Shuang Sun et al. This is an open access article distributed under the Creative Commons Attribution License, which permits unrestricted use, distribution, and reproduction in any medium, provided the original work is properly cited.

\begin{abstract}
This paper is intended to introduce a two-stage detection method to solve the multidamage problem in bridges. Vibration analysis is conducted to acquire the dynamic fingerprints which are regarded as information sources. Bayesian fusion is used to integrate these sources and preliminarily locate the damage. Then, the RSNB method which combines rough set theory and Naive-Bayes classifier is proposed to simplify the sample dimensions and fuse the remaining attributes for damage extent detection. A numerical simulation of a real structure, the Sishui Bridge in Shenyang, China, is conducted to validate the effectiveness of the proposed detection method. Data fusion based method is compared with single-valued index method at the damage localization stage. The proposed RSNB method is compared with the Back Propagation Neural Network (BPNN) method at the damage qualification stage. The results show that the proposed two-stage damage detection method has better performances in regard to transparency, accuracy, efficiency, noise robustness, and stability. Furthermore, an ambient excitation modal test was carried out on the bridge to obtain the vibration responses and assess the damage condition with the proposed method. This novel approach is applicable for early damage detection and provides a basis for bridge management and maintenance.
\end{abstract}

\section{Introduction}

Rapidly aging bridges are a serious problem in many countries. Bridges deteriorate gradually due to environmental effects and traffic loads, making them commonly structurally deficient over the course of their service life [1]. The early detection of damage is very significant to ensure bridge health and integrity, prevent catastrophic failure, and prolong their service life.

The vibration based damage detection method, as a kind of nondestructive evaluation technique, can be used to detect damage in bridges. Its basic principle is that damage causes changes in the bridge's structural properties which modify the vibration characteristics [2]. There are two general approaches of vibration based damage detection. First is the model updating approach, which involves updating certain parameters to ensure agreement between the experimentally measured modal parameters and the intact finite element model. The primary drawback of this method is that, due to the inevitable errors in modeling assumption and measurement, many models would reasonably match observations, which might lead to the wrong model identification [3]. The second most popular approach is the dynamic fingerprint method. Dynamic fingerprints are functions of the structural physical properties (mass, stiffness, and damping) and modal parameters (natural frequencies and mode shapes). Structural condition can be assessed through comparisons of the tested and intact responses. Although dynamic fingerprints are damage-sensitive they are limited in terms of application to civil infrastructures because the responses extracted from field sensors are readily influenced by environmental noise [4].

Recently, data fusion based damage detection method has attracted much attention [5-8]. Structural vibration responses are regarded as multiple sources and then transmitted to the fusion system. It can effectively solve the problems such as limited useful data and weak identification accuracy generally occurring in the field vibration data. Neural network, as a common data fusion technique, has been widely utilized by many researchers to identify structural damage. It mimics human cognitive processes to establish relations between input vibration responses and output damage condition. 
Papers that discuss neural networks on structural damage detection include, Yeung and Smith [8], Mehrjoo et al. [9], Jiang et al. [10], Min et al. [11], Shu et al. [12], Ugalde et al. [13], and Abdeljaber and Avci [14]. The basic and most important issue of this method is the training of a multilayer feed-forward neural network. A large number of neurons are needed to favor a better approximation, inevitably leading to a more complex system and higher computational cost. In neural networks, the mathematical approximation to describe the input and output relations is a black-box process. The disadvantage is the limited insights into the development of the model, which are hardly understandable by the decision maker [10].

In this work, we propose the incorporation of several fusion theories with recent developments in artificial intelligence to establish a transparent and efficient damage detection system. The proposed detection process begins by locating the damage through Bayesian fusion; then, damage extent is classified by RSNB method with the combination of rough set theory and Naive-Bayes classifier. To fully illustrate the detection performance of the proposed method, the damage location detection results by Bayesian fusion are compared with those by single-valued dynamic fingerprint. Damage extent detection by RSNB is compared with BPNN in terms of transparency, accuracy, efficiency, robustness, and stability. The overall organization of this paper is as follows: in Section 2, dynamic fingerprints are calculated to acquire damage information. In Section 3, the Bayesian fusion method is employed in order to achieve damage localization. In Section 4, a novel method called RSNB which makes use of rough set theory and Naive-Bayes classifier is presented for damage qualification. In Section 5, an example of application of the proposed method is provided. Conclusions are drawn in Section 5.

\section{Dynamic Fingerprints}

The structural damage can be detected by comparing the changes of dynamic fingerprint between the intact and damaged states at the measuring points.

2.1. Modal Flexibility Curvature (MFC). If any damage exists in a bridge, the stiffness will be reduced and cause a change of the flexibility because the structural stiffness matrix and flexibility matrix are reciprocal inversion matrices [15]. The flexibility matrix can be expressed as follows:

$$
\mathbf{F}=\sum_{k=1}^{m} \frac{1}{\omega_{k}^{2}} \phi_{k} \phi_{k}^{T}
$$

where $\omega_{k}$ is the $k$ th frequency; $\phi_{k}$ is the $k$ th mode shape vector; $m$ is the number of degree of freedoms (DOFs).

Modal flexibility difference can be written as follows:

$$
\Delta \mathbf{F}=\mathbf{F}_{u}-\mathbf{F}_{d}=\left[\begin{array}{cccc}
f_{11} & f_{12} & \cdots & f_{1 n} \\
f_{21} & f_{22} & \cdots & f_{2 n} \\
\vdots & \vdots & \ddots & \vdots \\
f_{n 1} & f_{n 2} & \cdots & f_{n n}
\end{array}\right]
$$

where $\mathbf{F}_{u}, \mathbf{F}_{d}$ are the intact and damaged modal flexibility matrices, respectively.

The maximum absolute values in each column of $\Delta \mathbf{F}$ are chosen to form a row matrix:

$$
\mathbf{f}=\left[\max \left(f_{j 1}\right) \max \left(f_{j 2}\right) \cdots \max \left(f_{j n}\right)\right] .
$$

Then the modal flexibility curvature is calculated by a central differentiation procedure. It can be expressed as the following [16]:

$$
\mathrm{MFC}=\frac{f(j+1)-2 f(j)+f(j-1)}{2 l^{2}},
$$

where $f(j)$ is the $j$ th element in the row matrix $\mathbf{f} ; l$ is the distance between two adjacent nodes.

2.2. Curvature Mode Shapes Difference (CMSD). Curvature mode shapes can be obtained from the mode shape displacement [17].

$$
\mathrm{CMS}=\frac{\phi_{k}(j+1)-2 \phi_{k}(j)+\phi_{k}(j-1)}{l^{2}},
$$

where $\phi_{k}(j)$ is the modal displacement at node $j$ of the $k$ th order; $l$ is the distance between two adjacent nodes.

The structural curvature mode shape difference of each order is given by the following:

$$
\mathrm{CMSD}=\mathrm{CMS}_{u}-\mathrm{CMS}_{d}
$$

where $\mathrm{CMS}_{u}$ and $\mathrm{CMS}_{d}$ are the intact and damaged CMS, respectively.

2.3. Uniform Load Surface Curvature Difference (ULSCD). Uniform load surface (ULS) is the derivative of modal flexibility [18]. For a linear system, the modal deflection at node $i$ under uniform unit load all over the structure can be approximated as the following:

$$
f(i)=\sum_{j=1}^{n} f_{i, j}=\sum_{k=1}^{m} \frac{\phi_{k}(i) \sum_{j=1}^{n} \phi_{k}(j)}{\omega_{k}^{2}},
$$

where $i, j$ represent the node numbers; $\phi_{k}(i)$ is the modal displacement at node $i$ of the $k$ th order; $m$ is the number of mode orders.

Based on the second-order difference principle, uniform load surface curvature can be given by the following:

$$
\mathrm{ULSC}=\frac{f(j+1)-2 f(j)+f(j-1)}{l^{2}},
$$

where $l$ is the distance between two adjacent nodes.

Uniform load surface curvature difference is defined as the following:

$$
\mathrm{ULSCD}=\mathrm{ULSC}_{u}-\mathrm{ULSC}_{d} \text {, }
$$

where $\mathrm{ULSC}_{u}, \mathrm{ULSC}_{d}$ are the intact and damaged ULSC, respectively. 


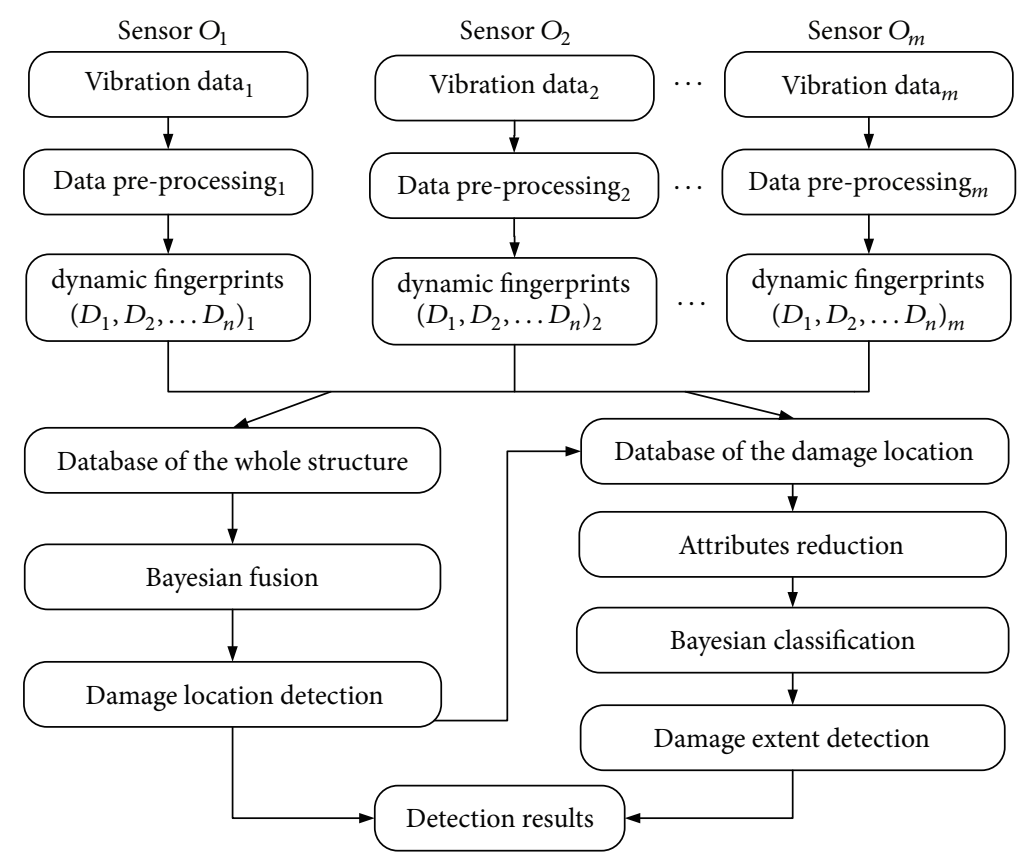

FIgURE 1: Two-stage structural damage detection system.

\section{Two-Stage Damage Detection System}

In this paper, a data fusion based damage detection system is set up. Dynamic fingerprints of every measurement point are sent to the fusion center. Then, a global decision is obtained to locate the damage. The fingerprints of the damaged objects are further used to detect the damage extent. The two-stage structural damage detection system is shown in Figure 1, and the main procedures are listed as follows:

(1) Calculate the dynamic fingerprints of each measurement point.

(2) Regard the dynamic fingerprints as information sources. Identify damage locations by Bayesian fusion.

(3) Constitute a decision table by the dynamic fingerprints of the suspected damage elements.

(4) Discretize the decision table. Reduce condition attributes by rough set theory.

(5) Train the Naive-Bayes classifier and identify the damage age extent.

3.1. Damage Localization Stage Based on Bayesian Fusion. In a damage detection system, suppose that $\mathrm{O}_{1}, \mathrm{O}_{2}, \ldots, \mathrm{O}_{m}$ represent the measurement sensors located in the suspected damage sites or elements to be identified. Information sources $S_{1}, S_{2}, \ldots, S_{n}$ represent the dynamic fingerprints.

The damage probability of element $O_{1}$ can be calculated according to Bayes' Theorem [19, 20]:

$$
\begin{aligned}
P\left(O_{1} \mid S\right) & =\frac{P\left(S \mid O_{1}\right) P\left(O_{1}\right)}{\sum_{i=1}^{m} P\left(S \mid O_{i}\right) P\left(O_{i}\right)} \\
& =\frac{\prod_{j=1}^{n} P\left(S_{j} \mid O_{1}\right) P\left(O_{1}\right)}{\sum_{i=1}^{m}\left[\prod_{j=1}^{n} P\left(S_{j} \mid O_{i}\right) P\left(O_{i}\right)\right]} .
\end{aligned}
$$

The prior probability $P\left(O_{i}\right)$ indicates the initial judgment of suspected damage locations before the extracted dynamic fingerprints are taken into account.

The conditional probability matrix can be expressed as follows:

$$
\left[\begin{array}{cccc}
P\left(S_{1} \mid O_{1}\right) & P\left(S_{2} \mid O_{1}\right) & \cdots & P\left(S_{n} \mid O_{1}\right) \\
P\left(S_{1} \mid O_{2}\right) & P\left(S_{2} \mid O_{2}\right) & \cdots & P\left(S_{n} \mid O_{2}\right) \\
\vdots & \vdots & \cdots & \vdots \\
P\left(S_{1} \mid O_{m}\right) & P\left(S_{2} \mid O_{m}\right) & \cdots & P\left(S_{n} \mid O_{m}\right)
\end{array}\right]
$$

where $P\left(S_{j} \mid O_{i}\right)$ is the probability value of extracted feature $S_{j}$ from the vibration data in sensor $O_{i}$.

$P\left(O_{i} \mid S\right)$ is the posterior probability; the certain damage sites are those with relatively high posterior probability values.

3.2. Damage Quantification Based on RSNB Method. In the second stage, we propose an effective identification method, RSNB, integrated with rough set and Naive-Bayes classifier. Rough set theory [21] is utilized to reduce the attributes of the original decision table formed by the suspected damage elements. Naive-Bayes [22] is utilized to fuse uncertain information in order to classify the damage extent.

3.2.1. Attribute Reduction by Rough Set Theory. In practice, there is often a great deal of measurement data with a considerable number of attributes. Rough set theory is utilized to reduce redundant features and increase efficiency. In the RSNB method, the dynamic fingerprints of the suspected damage elements are represented in the form of a decision table $S=(U, C \cup D, V, f)$. $U$ is the training or 
testing samples, condition attributes $C$ are dynamic fingerprints $\{$ MFC, 1st CMSD, 2nd CMSD, 3rd CMSD, ULSCD , decision attribute $D$ is the damage extent, $V$ represents a finite set of attribute values, and $f$ is an information function such that $f(x, a) \in V_{a}$ for every $a \in A, x \in U$.

Attribute reduction can only be applied to consistent decision tables. If all the objects with the same condition attribute belong to the same decision attribute, it is consistent; otherwise it is inconsistent. It can be expressed with $\operatorname{POS}_{C}(D)$ [23].

Given a decision table $S=(U, C \cup D, V, f), B \subseteq C$, the $B$-positive region of $D$ is

$$
\begin{aligned}
\operatorname{POS}_{B}(D) & =\bigcup_{X \in U / D} \underline{B}(X) \\
\underline{B}(X) & =\bigcup_{X_{I} \subseteq X} X_{i} \\
\bar{B}(X) & =\bigcup_{X_{i} \cap X \neq \varnothing} X_{i} \\
B_{B}(X) & =\bar{B}(X)-\underline{B}(X),
\end{aligned}
$$

where $\underline{B}(X)$ and $\bar{B}(X)$ are $B$-lower and $B$-upper approximations of $X$, respectively. $B N_{B}(X)$ is called $B$-boundary region of $X$, which represents the difference between $\underline{B}(X)$ and $\bar{B}(X)$. If $\operatorname{POS}_{C}(D)=U$, the decision table $S$ is consistent; otherwise $S$ is inconsistent. Inconsistent objects should be deleted before attribute reduction.

After the inconsistent objects are deleted, the heuristic algorithm based on attribute significance is utilized for attribute reduction [24].

The significance of attribute $a \in C-B$ is

$$
\begin{aligned}
\operatorname{SGF}(a, B, D) & =\gamma_{B \cup\{a\}}(D)-\gamma_{B}(D) \\
\gamma_{C}(D) & =\frac{\left|\operatorname{POS}_{C}(D)\right|}{|U|},
\end{aligned}
$$

where $\gamma_{C}(D)$ is the dependence between decision attribute $D$ and condition attributes $C$.

The process of the attribute reduction begins with a candidate for the reduct comprised of the entire set of condition attributes, which are removed in turn in increasing order according to significance SGF. If the dependence is unchanged, that is, $\gamma_{C-a}(D)=\gamma_{C}(D)$, then removed attribute $a$ can be deleted. Otherwise, it should be preserved. The final remaining attributes constitute the reduction set RED.

\subsubsection{Acquisition of Independent Variables by Rough Set The-} ory. In Naive-Bayes, the attributes $C_{1}, C_{2}, \ldots, C_{n}$ should all be conditionally independent of each other. The remaining attributes in the reduction set RED are independent and can be further used to build a Naive-Bayes classifier. The proof procedure is listed as follows.

In a decision table $S=(U, C \cup D, V, f), P$ and $Q$ are condition attributes, and $P \subseteq C, Q \subseteq C$.

(1) If $\operatorname{IND}(Q) \subseteq \operatorname{IND}(P)$, then $P$ is dependent on $Q, Q \Rightarrow$ $P, P$ can be deleted.

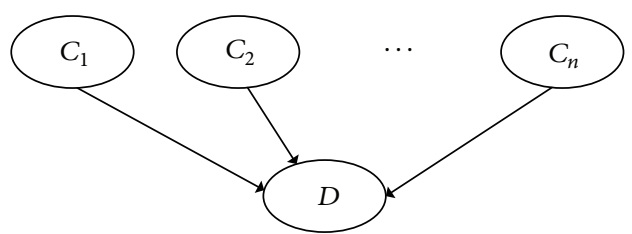

FIGURE 2: Naive-Bayes structure for damage extent detection with RSNB method.

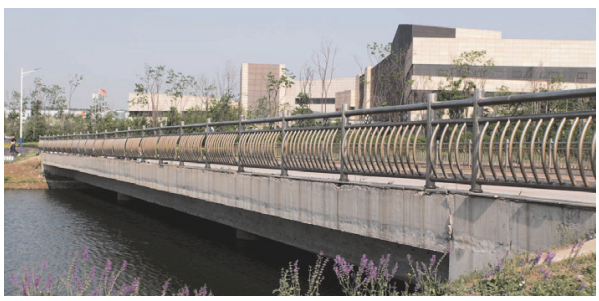

FIGURE 3: View of the Sishui Bridge.

(2) If $\operatorname{IND}(Q) \subseteq \operatorname{IND}(P)$ and $\operatorname{IND}(P) \subseteq \operatorname{IND}(Q)$, then $Q \Rightarrow P$ and $P \Rightarrow Q, P$ and $Q$ are equivalent.

(3) If $P$ is not dependent on $Q$, and $Q$ is not dependent on $P$, then $P$ and $Q$ are independent.

In a decision table $S=(U, C \cup D, V, f), R=\left(C_{1}, C_{2}\right)$ is a reduct of $C, R \subseteq C, \operatorname{POS}_{R}(D)=\operatorname{POS}_{C}(D)$; then $C_{1}, C_{2}$ are independent.

Reductio ad absurdum is used to verify the above proposition. It is assumed that if $C_{1}, C_{2}$ are dependent and $C_{2}$ is dependent on $C_{1}$, then $C_{1} \Rightarrow C_{2}, \operatorname{IND}\left(C_{1}\right) \subseteq \operatorname{IND}\left(C_{2}\right)$, $\operatorname{IND}\left(C_{1} \cup C_{2}\right)=\operatorname{IND}\left(C_{1}\right), \operatorname{POS}_{\left(C_{1} \cup C_{2}\right)}(D)=\operatorname{POS}_{C_{1}}(D)$, $\gamma_{\left(C_{1} \cup C_{2}\right)}(D)=\gamma_{C_{1}}(D), \operatorname{SGF}\left(C_{2}, C_{1}, D\right)=\gamma_{\left(C_{1} \cup C_{2}\right)}(D)-$ $\gamma_{C_{1}}(D)=0$; the attribute $C_{2}$ can be deleted, so the assumption is not reliable. $C_{1}, C_{2}$ cannot be dependent, so it can be concluded that the attributes in RED are all independent.

3.2.3. Damage Extent Classification by Naive-Bayes Classifier. After attribute reduction, an optimal decision table is obtained in which the columns are RED that consist of $n$ condition attributes $C_{1}, C_{2}, \ldots, C_{n}$ and one decision attribute $D$. Here, a Naive-Bayes network is applied to predict the damage extent (Figure 2).

When a set of new observed dynamic fingerprints $C_{\text {new }}=$ $\left(C_{1}, \ldots, C_{n}\right)$ arrives as the testing sample, the damage extent is predicted by the classification with the highest posterior probability. The damage classification rule is

$$
D_{k} \longrightarrow \max P\left(D=D_{k}\right) \prod_{i=1}^{n} P\left(C_{i} \mid D=D_{k}\right) \text {. }
$$

\section{Case Study}

4.1. Description of the Case Study Bridge. Sishui Bridge (Figure 3), which is located in Shenyang, China, is a concrete box-girder, continuous bridge with a main span of $20 \mathrm{~m}$ and dual $15 \mathrm{~m}$ side spans. Its width is $14 \mathrm{~m}$ and its height is $1.2 \mathrm{~m}$. (Figure 4). The bridge was constructed in 2009 and had been in service for seven years at the time this study was conducted. 


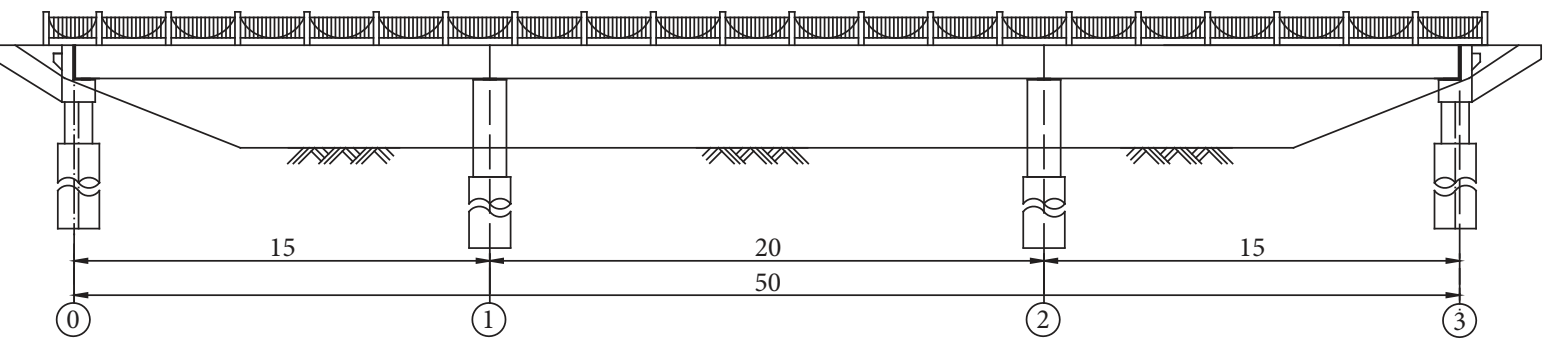

(a) Elevation drawing

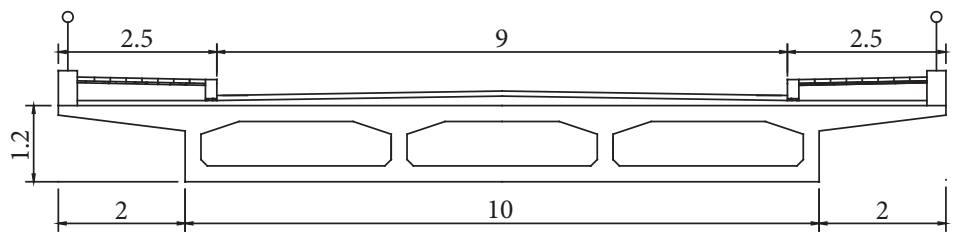

(b) Cross-section drawing

Figure 4: Sishui Bridge: elevation view and cross-section (unit: m).

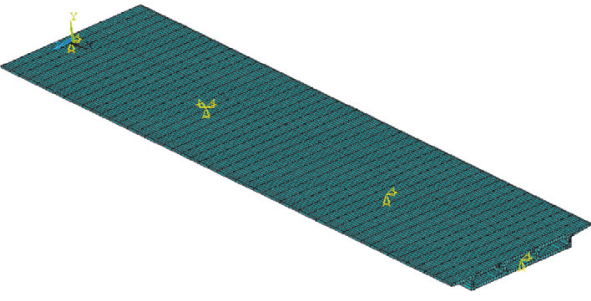

(a) Finite element model

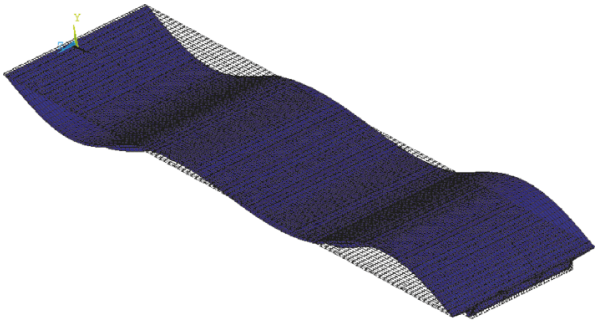

(c) Second mode shape

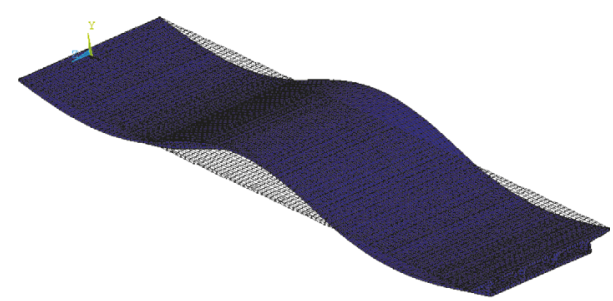

(b) First mode shape

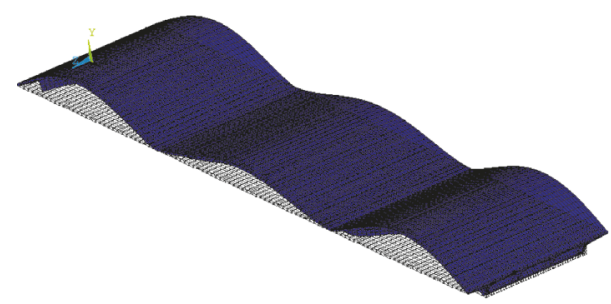

(d) Third mode shape

FIGURE 5: Finite element model and mode shapes of Sishui Bridge.

4.2. Numerical Simulation. Because it is impractical to apply physical damage to a real structure for the sake of analysis, a finite element model based on the bridge was built to simulate various damage scenarios. The model was then used to obtain the vibration properties in both the intact and damaged states and to thereby demonstrate the ability of the proposed twostage structural detection method. The bridge was modeled with the BEAM 188 element, where each $0.5 \mathrm{~m}$ along the bridge's length requires 101 nodes and 100 elements. The mass density is $2600 \mathrm{~kg} / \mathrm{m}^{3}$ and the elastic modulus is $32.5 \mathrm{GPa}$. The finite element model of the bridge is shown in Figure 5(a).

Damage in the numerical model can be simulated by reducing Young's modulus of the specified elements [25]. Damage elements are assumed to be located near the middle of the three spans (elements nos. 15, 50, and 86). For the intact bridge, the natural frequencies of the first three orders are $7.4894 \mathrm{~Hz}, 11.636 \mathrm{~Hz}$, and $13.03 \mathrm{~Hz}$; the mode shapes are shown in Figures 5(b)-5(d).

4.2.1. Influence of Data Discretization in RSNB. The rough set is just available to deal with discrete values. The aim of discretization is to classify the scope of continuous values into finite small intervals and then regard them as categorical features. The discretization course should make no change of the former indiscernible relation. In this paper, $k$-means clustering algorithm is used to discretize attribute values [26]. The discretization level or number of clusters for partition intervals critically influences the classification accuracy. The optimal selection of the number of clusters $k$ is studied in the following. 
TABLE 1: Detection results with RSNB method when $n=2$ (classification: $5 \%$ and $10 \%$ damage).

\begin{tabular}{ccccc}
\hline$k$ & Consistent & Prior probabilities & NA & IA \\
\hline 2 & Yes & $1.00,1.00$ & 2 & 1.000 \\
3 & Yes & $1.00,1.00$ & 2 & 1.000 \\
4 & Yes & $1.00,1.00$ & 5 & 0.955 \\
5 & Yes & $1.00,1.00$ & 5 & 0.955 \\
6 & Yes & $1.00,1.00$ & 5 & 0.960 \\
7 & Yes & $1.00,1.00$ & 5 & 0.950 \\
\hline
\end{tabular}

TABLE 2: Detection results with RSNB method when $n=3$ (classification: $5 \%, 10 \%$, and $15 \%$ damage).

\begin{tabular}{ccccc}
\hline$k$ & Consistent & Prior probabilities & NA & IA \\
\hline 2 & No & $0.96,0.96,1.00$ & 10 & 0.950 \\
3 & Yes & $1.00,1.00,1.00$ & 2 & 1.000 \\
4 & Yes & $1.00,1.00,1.00$ & 2 & 1.000 \\
5 & Yes & $1.00,1.00,1.00$ & 6 & 0.963 \\
6 & Yes & $1.00,1.00,1.00$ & 6 & 0.967 \\
7 & Yes & $1.00,1.00,1.00$ & 5 & 0.957 \\
\hline
\end{tabular}

TABLE 3: Detection results with RSNB method when $n=4$ (classification: 5\%, 10\%, 15\%, and 20\% damage).

\begin{tabular}{ccccc}
\hline$k$ & Consistent & Prior probabilities & NA & IA \\
\hline 2 & No & $0.00,0.59,0.90,0.99$ & $/$ & $/$ \\
3 & No & $0.97,0.97,1.00,1.00$ & 10 & 0.980 \\
4 & Yes & $1.00,1.00,1.00,1.00$ & 2 & 1.000 \\
5 & Yes & $1.00,1.00,1.00,1.00$ & 2 & 1.000 \\
6 & Yes & $1.00,1.00,1.00,1.00$ & 6 & 0.968 \\
7 & Yes & $1.00,1.00,1.00,1.00$ & 6 & 0.965 \\
\hline
\end{tabular}

TABLE 4: Detection results with RSNB method when $n=5$ (classification: $5 \%, 10 \%, 15 \%, 20 \%$, and $25 \%$ damage).

\begin{tabular}{ccccc}
\hline$k$ & Consistent & Prior probabilities & NA & IA \\
\hline 2 & No & $0.00,0.06,0.94,0.40,0.00$ & $/$ & $/$ \\
3 & No & $0.78,0.91,0.98,0.98,1.00$ & 9 & 0.934 \\
4 & No & $0.97,0.99,1.00,1.00,1.00$ & 8 & 0.978 \\
5 & Yes & $1.00,1.00,1.00,1.00,1.00$ & 2 & 1.000 \\
6 & Yes & $1.00,1.00,1.00,1.00,1.00$ & 2 & 1.000 \\
7 & Yes & $1.00,1.00,1.00,1.00,1.00$ & 4 & 0.998 \\
\hline
\end{tabular}

With regard to the noise level $p=15 \%, 6$ kinds of damage patterns $(5 \%, 10 \%, 15 \%, 20 \%, 25 \%$, and $30 \%)$ are studied. The detection results with different clusters are listed in Tables $1-5$. Figure 6 shows the influences of " $k$ " on $I A$ for every classification under $15 \%$ noise.

From the results listed in Tables 1-5, it can be observed that when $k<n$, the decision table is inconsistent. Inconsistent objects should be deleted which might decrease the prior probabilities and further influence IA.

When $k=n-1$, only a small portion of objects needs to be deleted. That means it will not greatly affect the prior probability. The number of remaining attributes is around 10
TABLE 5: Detection results with RSNB method when $n=6$ (classification: $5 \%, 10 \%, 15 \%, 20 \%, 25 \%$, and $30 \%$ damage).

\begin{tabular}{ccccc}
\hline$k$ & Consistent & Prior probabilities & NA & IA \\
\hline 2 & No & $0.00,0.00,0.41,0.92,0.01,0.00$ & $/$ & $/$ \\
3 & No & $0.00,0.44,0.94,0.97,0.91,0.96$ & $/$ & $/$ \\
4 & No & $0.90,0.91,1.00,1.00,1.00,1.00$ & 10 & 0.965 \\
5 & No & $0.99,0.98,1.00,1.00,1.00,1.00$ & 9 & 0.983 \\
6 & Yes & $1.00,1.00,1.00,1.00,1.00,1.00$ & 2 & 1.000 \\
7 & Yes & $1.00,1.00,1.00,1.00,1.00,1.00$ & 2 & 1.000 \\
\hline
\end{tabular}

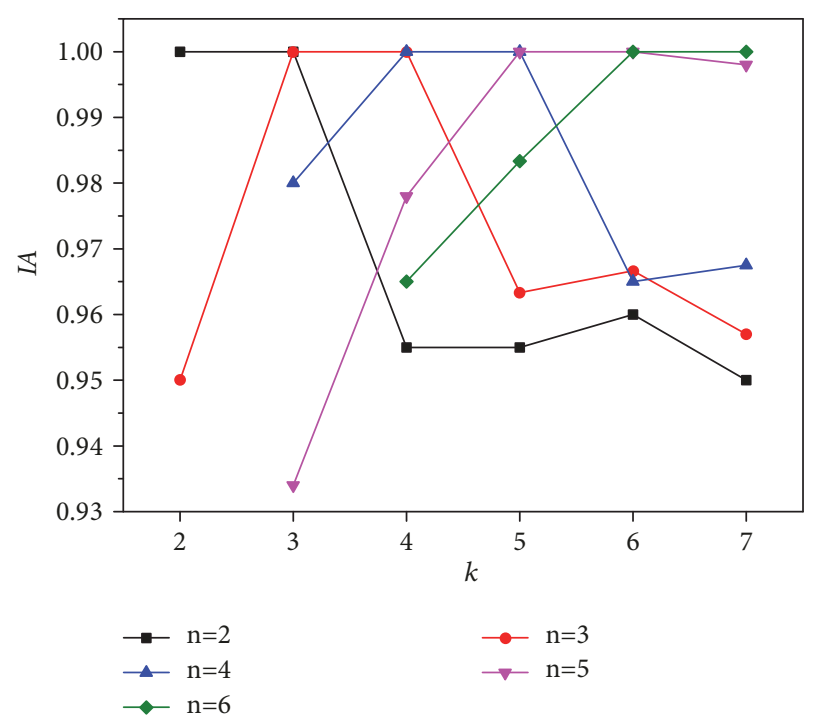

Figure 6: Comparison of identification accuracy with different number of clusters.

when compared to 15 of the original table. This means that $1 / 3$ of the attributes can be reduced. Therefore, the results are acceptable when $k=n-1$.

When $k<n-1$, a massive number of objects need to be deleted. The great loss of objects will greatly influence the prior probability and sometimes a pattern will disappear and cannot go to the next step for classification (e.g., in Table 3, $n=4, k=2$ ). Consequently, the RSNB method cannot be applied when $k<n-1$.

When $k \geq n$, the decision table is consistent and the prior probability cannot be changed.

When $k=n$ or $k=n+1$, the number of remaining attributes is around 2 when compared to 15 of the original table. Then the storage space of the decision table is released to the minimum. The identification accuracy "IA" is close to $100 \%$. Therefore, we can achieve the optimal results when $k=n$ or $k=n+1$.

When $k>n+1$, the number of remaining attributes is around 5 when compared to 15 of the original table. The lowest IA is $95 \%$, as shown in Table 1 . The highest IA is $99.8 \%$, as shown in Table 4.

From Figure 6, different numbers of clusters ranging from 2 to 7 were tested. The best results are obtained when the number of clusters is equal to the number of patterns, that is, $k=n$. When $k>n$, the IA remained $100 \%$ at $k=n+1$ and 


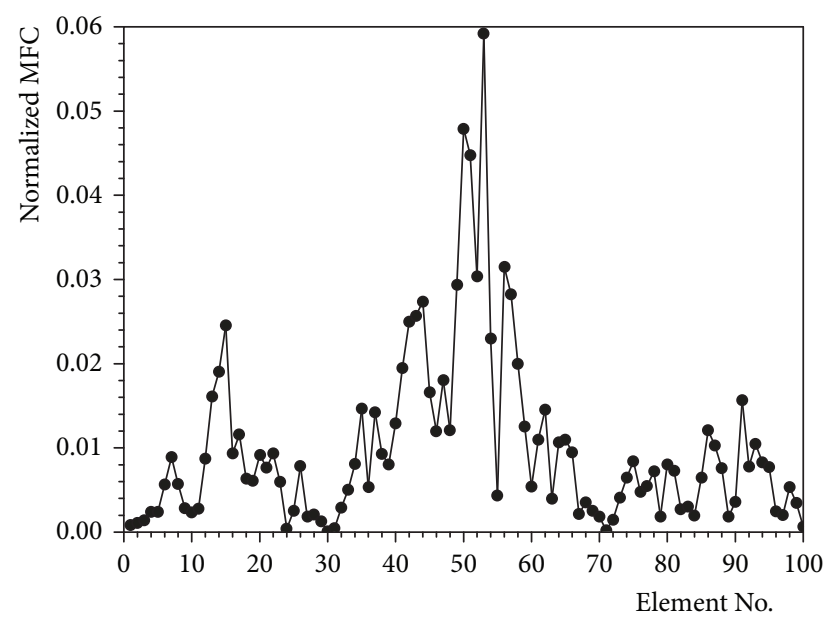

Figure 7: Damage localization with MCF.

then gradually decreased. When $k<n$, IA rapidly dropped and sometimes the damage patterns cannot be classified. However, the results are still acceptable if $k=n-1$.

4.2.2. Comparison between Data Fusion and Single Dynamic Fingerprint. To verify the ability of data fusion based damage detection method, the fusion damage localization results are compared with those obtained via single dynamic fingerprint. It is assumed that $5 \%$ damage occurs in elements 15,50 , and 86. The first three frequencies and vertical mode shapes of the intact and damaged states are acquired from the numerical model. In order to compare the noise robustness of the two methods, $15 \%$ noise is added to the mode shapes [27]. Five dynamic fingerprints MFC, 1st CMSD, 2nd CMSD, 3rd CMSD, and ULSCD of each element are calculated by (1)-(9) and normalized into probability values for Bayesian fusion.

Damage location detection is based on Bayes' Theorem (see (10)); objects $O_{1}, O_{2}, \ldots, O_{m}$ represent the damage sites or elements to be identified. Five information sources $S_{1}$ to $S_{5}$ represent the MFC, 1st CMSD, 2nd CMSD, 3rd CMSD, and ULSCD, respectively. As there is no prior information of initial drawback of the bridge, the concrete of the beam is assumed to be homogeneous isotropic material, the prior probability of each element is equivalent, and $P\left(O_{i}\right)=$ $1 / 100(i=1,2, \ldots, 100)$. The elements with relatively higher posterior probability values are identified as damage sites.

The fusion damage localization results under $15 \%$ noise are compared with those obtained via single normalized dynamic fingerprint method as shown in Figures 7-12.

As shown in Figures 7-11, under 15\% noise, we found that the identification results undergo a large amount of interference and that correct damage locations are not possible to be obtained with only a single dynamic fingerprint method. After Bayesian fusion, the probability values of the three damage elements are apparently higher; their fusion probabilities are $0.14,0.35$, and 0.27 , respectively. The values of other elements do not exceed 0.05 and are nearly 0 , so the Bayesian fusion method is more sensitive to damage and more resistant to noise. It can also effectively reduce the

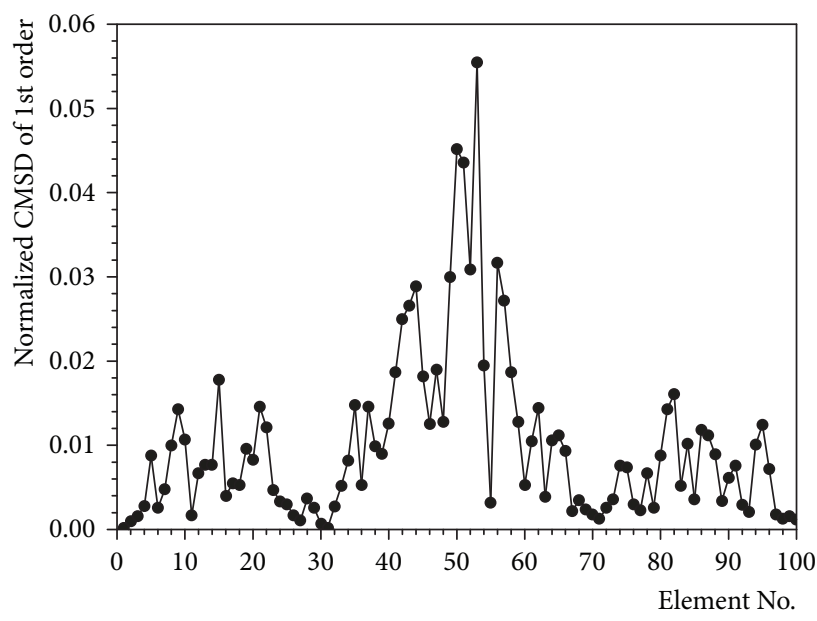

FIGURE 8: Damage localization with 1st CMSD.

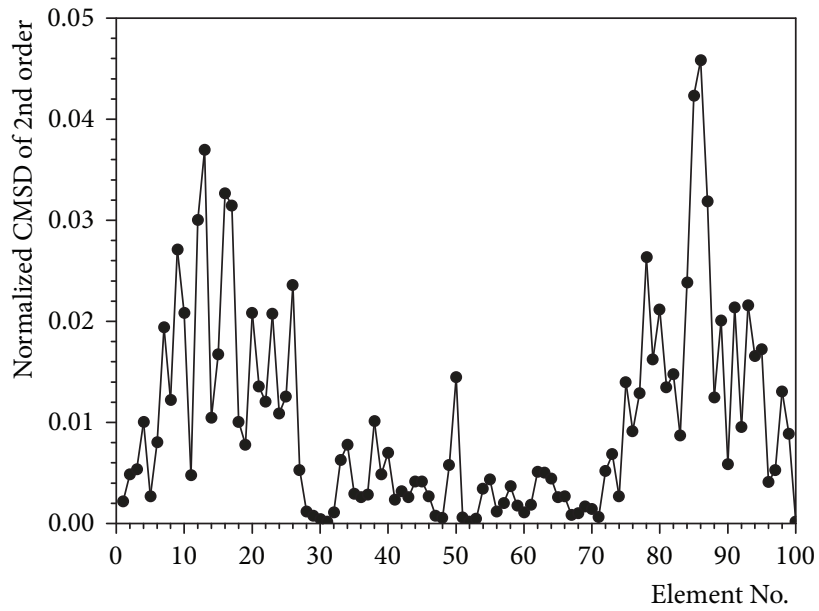

FIgure 9: Damage localization with 2nd CMSD.

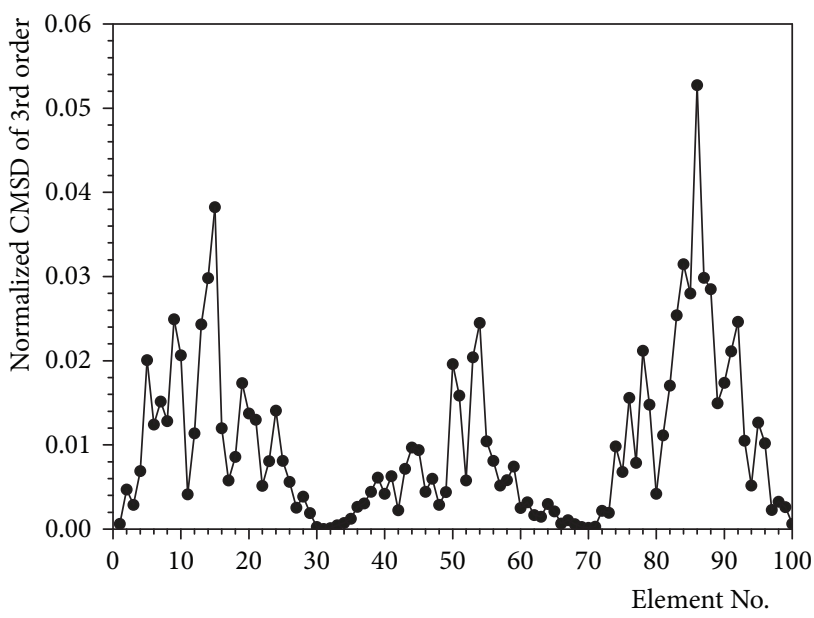

FIgure 10: Damage localization with 3rd CMSD.

number of potential damage sites identified. In particular, this method can localize the damage sites even if the damage is $5 \%$, it can realize early detection of structural failure. 


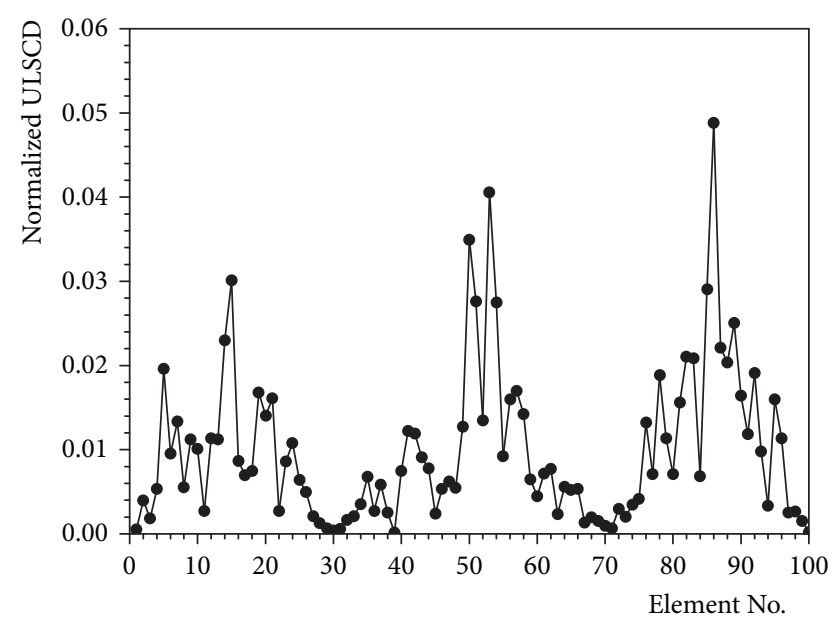

FIGURE 11: Damage localization with ULSCD.

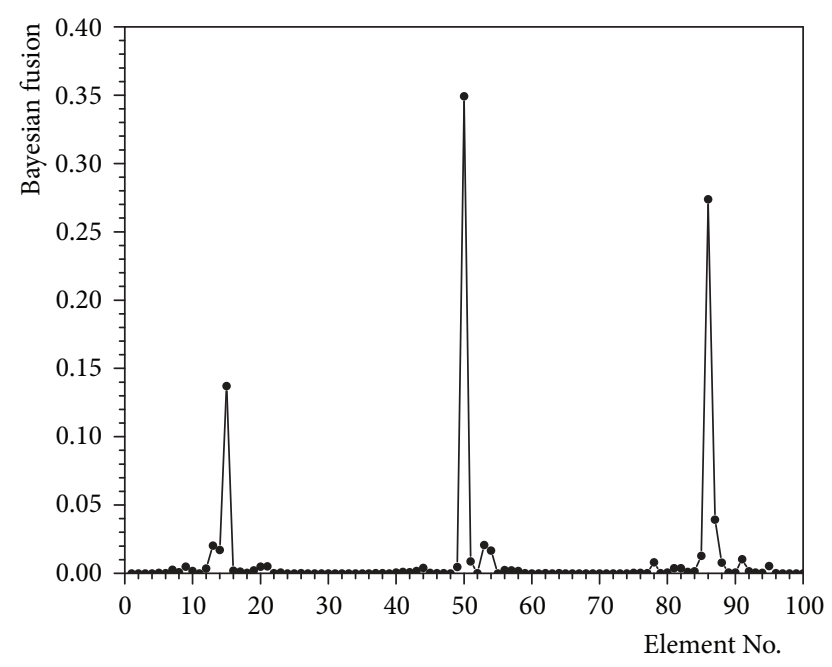

FIgURE 12: Damage localization with Bayesian fusion.

4.2.3. Comparison between BPNN and RSNB. After the localization stage, three suspected elements (elements nos. 15,50 , and 86) are extracted for damage extent detection. Two kinds of fusion methods are applied to compare the identification results. One is the proposed RSNB method; the other is Back Propagation Neural Network (BPNN). They are compared in terms of system transparency, identification accuracy, efficiency, noise robustness, and stability under small sample sizes.

(1) System Transparency. For the BPNN method, as shown in Figure 13, a three-layer neural network is selected: 15 neurons in the input layer indicate the dynamic fingerprints of the damage elements which have been identified at the localization stage; 1 neuron in the output layer indicates the damage extent; 30 neurons are in the hidden layer (twice as the neurons in the input layer). The other parameters are determined as follows: learning rate $\mathrm{lr}=0.1$; error goal $=$ 0.0000005; and maximum iterations Epoch $=400$. The hidden layer of BPNN is treated as a black-box; the process of neural networks often manifests in an unclear way that does not allow people to retrieve and understand the exact relationship between the provided information and the final decision. Moreover, it is well known that, due to their strong nonlinear behavior, the neural networks require a large number of neurons to deal with complex systems, which will lead to a more complex model and higher computational cost.

For the RSNB method (Figure 14), 15 input dynamic fingerprints are regarded as the original condition attributes. The optimal number of clusters $k$ for data discretization can be determined according to Section 4.2.1. Compared with the BPNN method, the proposed RSNB manifests in a clear way for damage extent identification. The attribute reduction process involves calculating the significance SGF of every condition attribute. The Naive-Bayes classifier obtains the final classification through calculating the prior and conditional probabilities. Hence, the whole system of the RSNB method is absolutely transparent. Therefore, the decision maker can fully control its origin and meaning, which is encouraged to apply on the damage detection.

(2) Identification Accuracy, Efficiency, Noise Robustness, and Stability. In the following, four damage patterns $(5 \%, 10 \%$, $15 \%$, and $20 \%$ ) are examined under various noise levels to compare the detection ability of the two methods in regard to accuracy, efficiency, and robustness.

To compare the robustness of BPNN and RSNB to variations in sample size and noise level, the identification accuracy (IA) and runtime of the two methods when $n=4$ are shown in Figures 15 and 16. From these results, the following phenomena can be observed.

Firstly, with the BPNN method, IA decreases as the noise level " $p$ " increases. It is shown that when $N=50$, the decline rate of IA is $31.1 \%$ (from $96.5 \%$ to $66.5 \%$ ). However, although the noise level increases, the IA with the RSNB method is still as high as $100 \%$. This indicates that the RSNB method has better antinoise capacity than the BPNN method.

Secondly, it can be observed that the BPNN method cannot get an ideal detection result if the sample size is not large enough. The BPNN algorithm needs enough training samples to adjust the weighted-coefficient and threshold values in order to establish the relations between parameters. From Figure 15, the IA with BPNN drops a lot under a small sample size such as $N=50$ or 75 . Therefore, the IA is obviously lower when $N=50$ or 75 than $N=100$ or 200 . Nevertheless, the IA with RSNB method is not influenced by the sample size. Even if $N=50$, it can still get a high IA value. The relationship between the computational cost and the sample size can also be revealed. In BPNN, the runtime rises as the sample size increases. However, the runtime values did not have much change in RSNB. This is due to the fact that calculating the conditional probabilities in RSNB is more efficient than nonlinear learning in BPNN.

Thirdly, under the same noise level and sample size, the RSNB method possesses a better damage detection capacity in accuracy and efficiency. Even if $N=50, p=25 \%$, the IA is as high as $100 \%$ in RSNB compared with $66.5 \%$ in BPNN. As the sample size enlarges, when $N=200$, the maximum runtime is $0.0589 \mathrm{~s}$. This is almost the same as $0.0581 \mathrm{~s}$ when 


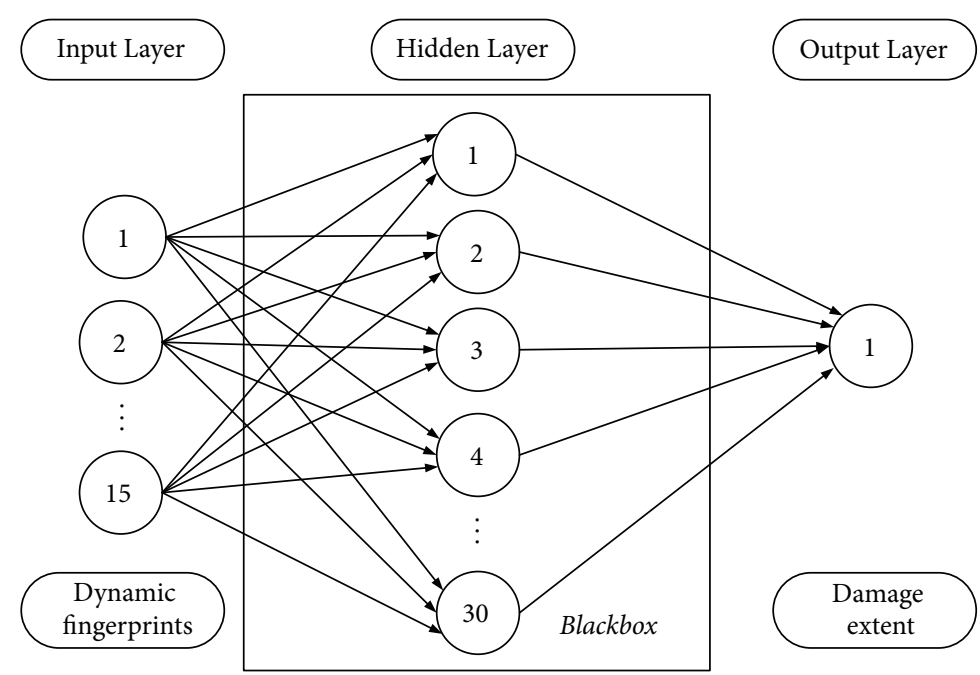

FIgURE 13: Topological structure of a three-layer BPNN method.

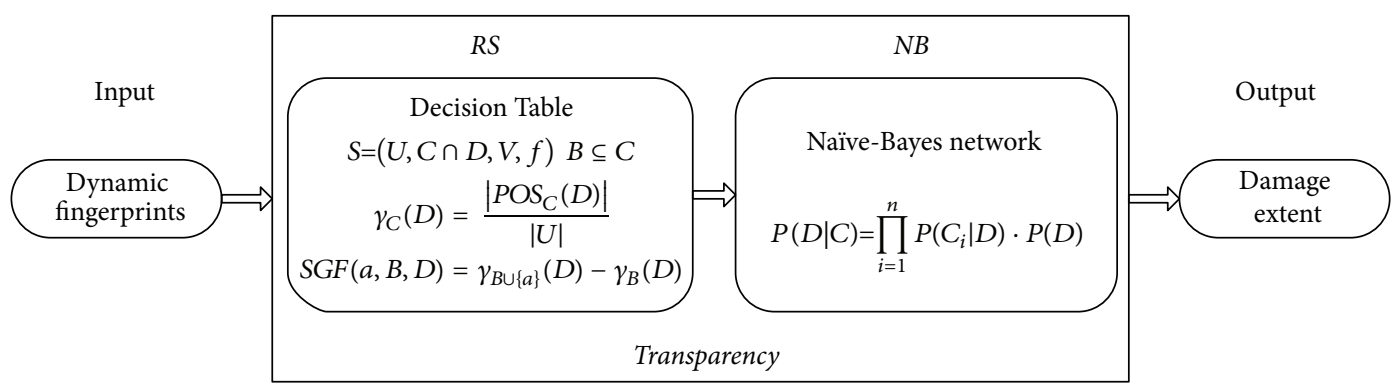

FIGURE 14: Topological structure of RSNB method.

\begin{tabular}{|c|c|c|c|c|}
\hline \multicolumn{5}{|c|}{ RSNB } \\
\hline$N$ & $I A(\%)$ & & Runtimes & (s) \\
\hline 50 & 100 & Good & 0.0581 & Good \\
\hline 75 & 100 & & 0.0586 & \\
\hline 100 & 100 & & 0.0571 & \\
\hline 200 & 100 & Good & 0.0589 & Good \\
\hline
\end{tabular}

\begin{tabular}{|cc|cr|c|}
\hline \multicolumn{5}{|c|}{ BPNN } \\
$N$ & $I A(\%)$ & \multicolumn{2}{|c|}{ Runtimes (s) } \\
\hline 50 & 66.5 & & 0.446 & \\
75 & 82.0 & & 0.625 & \\
100 & 96.5 & & 1.878 & \\
200 & 99.4 & Goor & 2.508 & \\
\hline
\end{tabular}

Figure 15: Comparison of RSNB and BPNN in different sample sizes ( $p=25 \%$ ).

\begin{tabular}{|c|c|c|c|c|}
\hline \multicolumn{5}{|c|}{ RSNB } \\
\hline$p$ & $I A(\%)$ & & Runtimes & \\
\hline $10 \%$ & 100 & Good & 0.0890 & Good \\
\hline $15 \%$ & 100 & & 0.0866 & \\
\hline $20 \%$ & 100 & & 0.0872 & \\
\hline $25 \%$ & 100 & Good & 0.0589 & Good \\
\hline
\end{tabular}

\begin{tabular}{|c|c|c|c|c|}
\hline \multicolumn{5}{|c|}{ BPNN } \\
\hline$p$ & $I A(\%)$ & $\mathrm{R}$ & ntimes & \\
\hline $10 \%$ & 96.5 & Good & 0.487 & Poor \\
\hline $15 \%$ & 95.5 & & 0.568 & \\
\hline $20 \%$ & 90.0 & & 0.509 & \\
\hline $25 \%$ & 66.5 & Poor & 0.446 & Poor \\
\hline
\end{tabular}

FIGURE 16: Comparison of RSNB and BPNN in different noise levels $(N=50)$. 


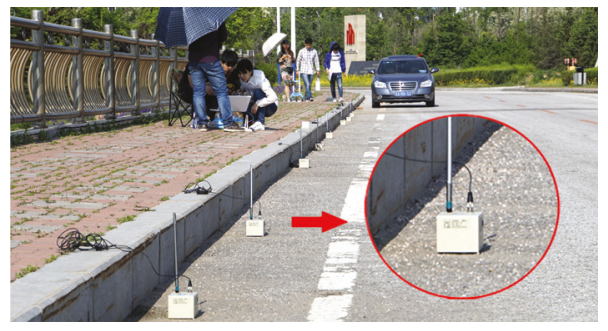

FIGURE 17: Velocity sensors in the ambient test.

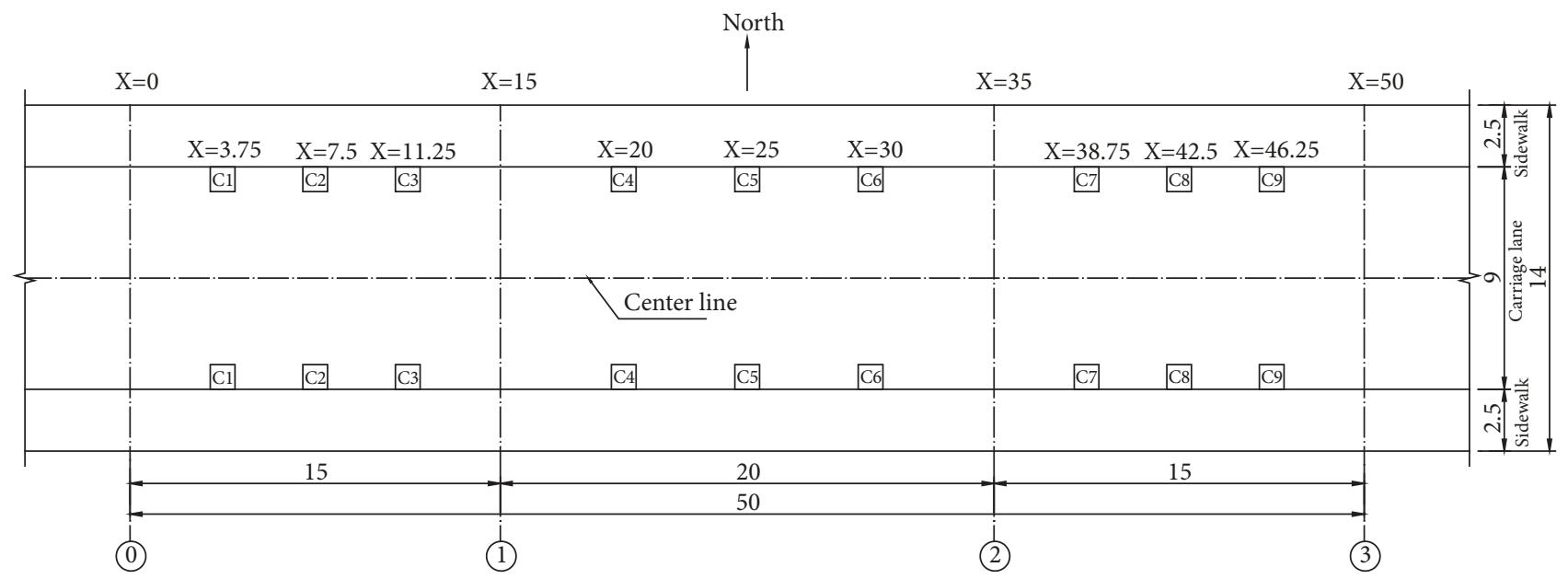

FIGURE 18: Positions of measurement points.

TABLE 6: Comparison of the two methods.

\begin{tabular}{lcc}
\hline Aspect & BPNN & RSNB \\
\hline Identification accuracy & Medium & High \\
Computational cost & Low & High \\
Acceptability & Black-box & Transparency \\
Noise robustness & Poor & Good \\
Stability under small sample sizes & Poor & Good \\
\hline
\end{tabular}

$N=50$ in RSNB. As a contrast, in BPNN, when $N=200$, the maximum runtime is $2.508 \mathrm{~s}$. This is almost six times as much as the cost of $0.446 \mathrm{~s}$ when $N=50$. This implies the RSNB method performs more stably under high noise level and small sample size than BPNN method.

In conclusion, the advantages and drawbacks of each method are summarized in Table 6.

\subsection{Ambient Excitation Modal Test}

4.3.1. Experimental Procedures. An ambient test was carried out on Sishui Bridge on May 19, 2017, to verify the applicability of the RSNB method. The pieces of equipment required in this test are as follows: velocity sensor DH5907 A module (wireless), modem, large capacity power bank, WiFi, and GPS. The frequency range of the DH5907A module is $0.12 \mathrm{~Hz} \sim 78 \mathrm{~Hz}$. The velocity sensors used in this ambient test are shown in

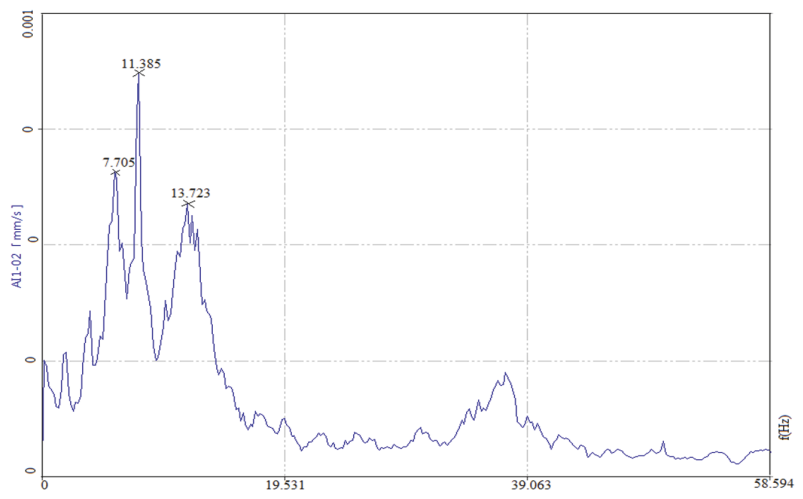

FIGURE 19: Spectrum curve.

Figure 17. Nine sensors were selected to be placed on quarter and half of each span of the bridge (Figure 18).

The response of the bridge due to ambient vibration is analyzed by Dong Hua Mode Analysis (DHMA) software. After spectrum analysis, the natural frequencies can be identified by larger peaks of the spectrum curve shown in Figure 19. The comparison of frequency and mode shape results from ambient test and numerical simulation is illustrated in Table 7 and Figure 20, respectively. In order to show the changes obviously, the mode shapes are normalized to unity. 


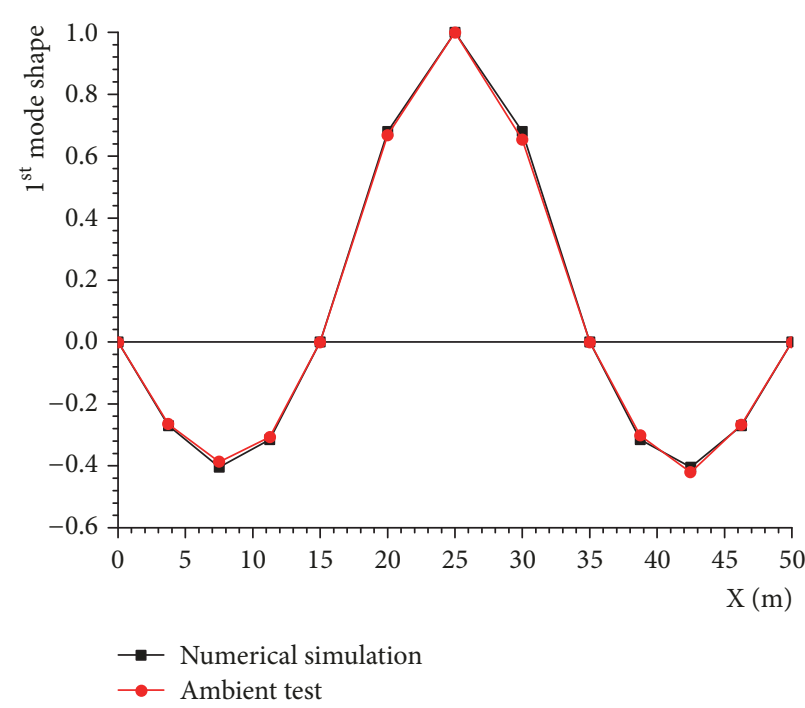

(a) First mode shape

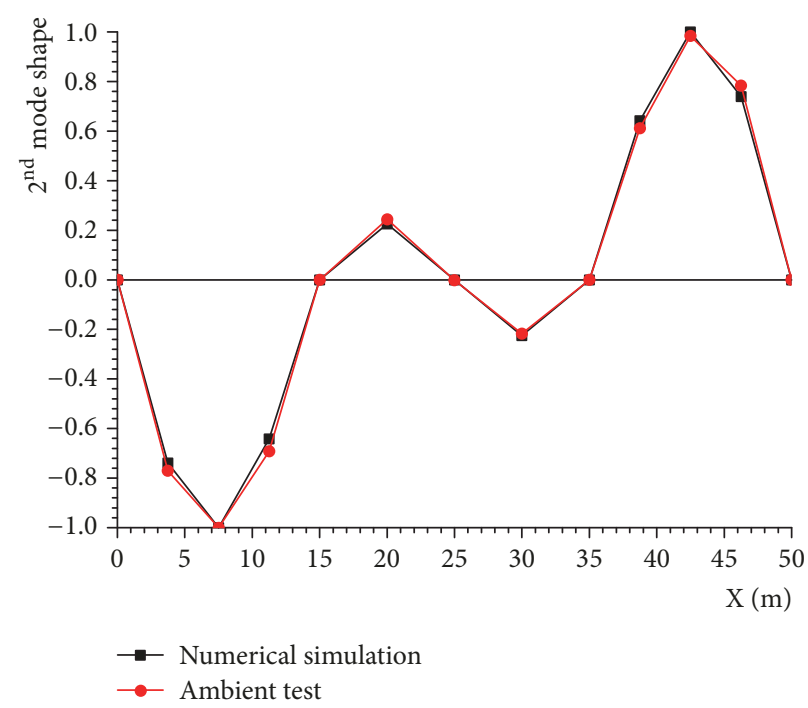

(b) Second mode shape

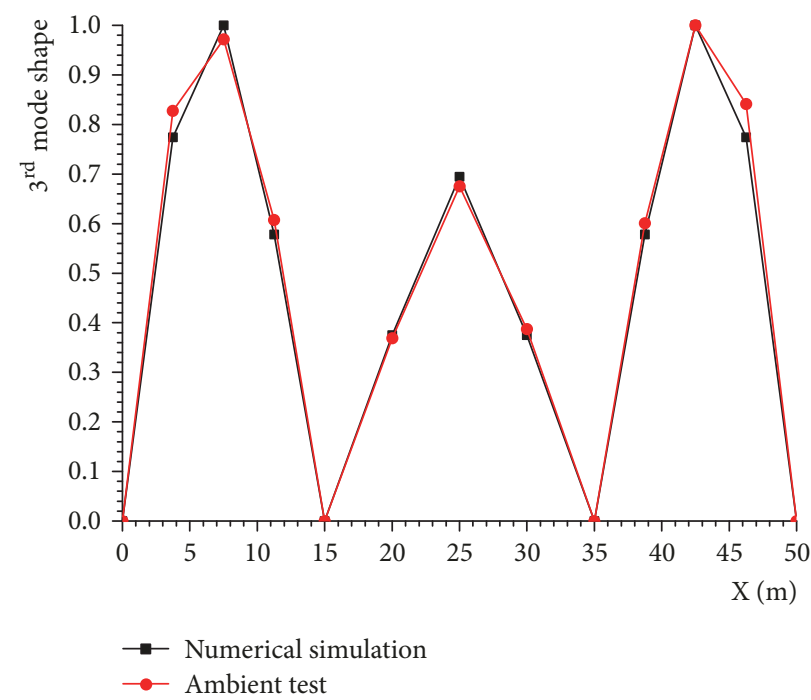

(c) Third mode shape

FIgURE 20: Mode shapes of numerical simulation and ambient test.

TABLE 7: Frequencies of numerical simulation and ambient test.

\begin{tabular}{lccc}
\hline Solution methods & $\begin{array}{c}\text { st mode } \\
(\mathrm{Hz})\end{array}$ & $\begin{array}{c}\text { 2nd mode } \\
(\mathrm{Hz})\end{array}$ & $\begin{array}{c}\text { 3rd mode } \\
(\mathrm{Hz})\end{array}$ \\
\hline Numerical simulation & 7.4894 & 11.636 & 13.03 \\
Ambient test & 7.705 & 11.385 & 13.723 \\
\hline
\end{tabular}

4.3.2. Analysis Results. Since a limited number of sensors are utilized in practice, the mode shapes of the 9 measurement points are refined by spline curve fitting [28]. The dynamic fingerprints MFC, CMSD, and ULSCD are calculated according to (1)-(9) and then regarded as information sources and fused according to (10). The damage location detection result of the 9 points is illustrated in Figure 21. After Bayesian fusion, the probability values of measurement points 2 and
5 are apparently higher and they are identified to be the suspected damage elements.

Then the damage extent of Sishui Bridge is assessed with the RSNB method; the process is shown in Figure 22. The damage pattern of the bridge is estimated to be $5 \%, 10 \%$, and $15 \%$. As described in Section 4.2.1, when the number of patterns $n=3$, the optimal number of clusters is $k=3$ for data discretization. Instead of extracting all the vibration indexes, only 2 remaining attributes are required for damage classification; they are 2nd CMSD of points 2 and 5; the two values are considered as a testing sample and sent to the Naive-Bayes classifier. The classification results are $100 \%, 0 \%$, and $0 \%$ corresponding to the damage levels $5 \%, 10 \%$, and $15 \%$. Thus, the damage extent is identified to be around $5 \%$.

The damage level of Sishui Bridge is assessed to be small. Routine damage detection is suggested for the bridge to avoid further deterioration. 


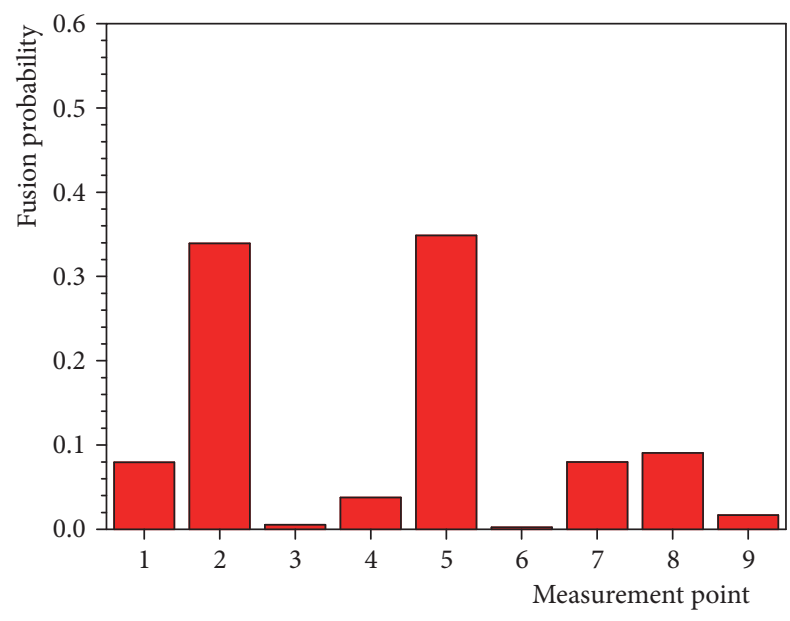

FIGURE 21: Damage localization results.

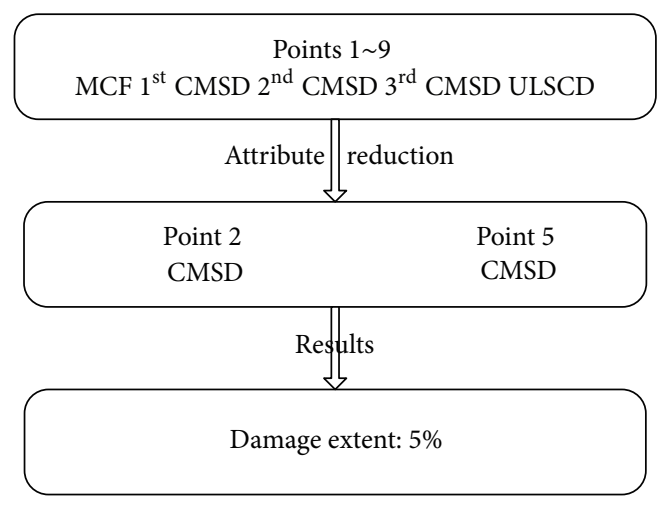

FIGURE 22: Damage qualification results.

\section{Conclusion}

This paper presented a novel damage detection method of bridges. At the damage localization stage, dynamic fingerprints calculated by the vibration responses are fused via Bayes' Theorem to identify the damage sites. At the damage quantification stage, an innovative approach based on rough set theory and the Naive-Bayes classifier is introduced as a highly interpretable and efficient method. The most notable conclusions of this study can be summarized as follows:

(1) Bayesian fusion is more effective in resisting noise and locating damage than the use of individual dynamic fingerprints. The localization stage can minimize the potential damage sites and enhances the calculation efficiency.

(2) The $k$-means clustering algorithm is utilized for data discretization. The number of clusters $k$ impacts the identification accuracy. The sample simplicity and identification accuracy are optimal when $k=n$ or $k=n+1$. The result when $k=n-1$ is still acceptable, though some objects are deleted for the sake of inconsistency in the decision table.

(3) Naive-Bayes classifier is simple in structure and quick to calculate; however, it has a rigorous restriction that each variable should all be conditionally independent of its nondescendants. The reduction property of rough set theory can reduce sample dimensions and acquire independent variables for Naive-Bayes. Compared to the BPNN method, the RSNB lends advantages in transparency, accuracy, efficiency, noise robustness, and stability under small sample sizes.

(4) An ambient excitation modal test was carried out on the bridge to validate the applicability of the proposed method. The damage extent of Sishui Bridge is classified to be $5 \%$; routine damage detection is suggested to avoid further deterioration. The bridge type selected for the case study is common throughout China in terms of its span and structural details, suggesting that the results obtained here could be replicated with a large number of existing bridges.

\section{Notations}

$\begin{array}{ll}N: & \begin{array}{l}\text { Number of training (testing) samples of } \\ \text { each pattern }\end{array} \\ n: & \text { Number of patterns } \\ p: & \text { Noise level } \\ k: & \text { Number of clusters } \\ \text { IA: } & \text { Average ratio of correct identification } \\ & \text { samples to total samples of } n \text { patterns } \\ \text { NA: } & \text { Number of remaining attributes } \\ \text { RED: } & \text { Reduction set } \\ \text { lr: } & \text { Learning rate } \\ \text { Epoch: } & \text { Maximum iterations. }\end{array}$

\section{Conflicts of Interest}

The authors declare that there are no conflicts of interest regarding the publication of this article.

\section{Acknowledgments}

This work is supported by the National Natural Science Foundation of China (Grant no. 51474048) and the Fundamental Research Funds for the Central Universities (N170104024).

\section{References}

[1] J. Wasowski, F. Bovenga, R. Nutricato, D. O. Nitti, and M. T. Chiaradia, "High resolution satellite multi-temporal interferometry for monitoring infrastructure instability hazards," Innovative Infrastructure Solutions, vol. 2, no. 1, 2017.

[2] Y. Bao, C. Song, W. Wang, T. Ye, L. Wang, and L. Yu, "Damage detection of bridge structure based on SVM," Mathematical Problems in Engineering, vol. 2013, Article ID 490372, 2013.

[3] Y. Robert-Nicoud, B. Raphael, and I. F. C. Smith, "System identification through model composition and stochastic search," Journal of Computing in Civil Engineering, vol. 19, no. 3, pp. 239247, 2005.

[4] F. N. Catbas, M. Susoy, and D. M. Frangopol, "Structural health monitoring and reliability estimation: long span truss bridge application with environmental monitoring data," Engineering Structures, vol. 30, no. 9, pp. 2347-2359, 2008.

[5] M. M. R. Taha and J. Lucero, "Damage identification for structural health monitoring using fuzzy pattern recognition," Engineering Structures, vol. 27, no. 12, pp. 1774-1783, 2005. 
[6] P.-H. Chen, H.-K. Shen, C.-Y. Lei, and L.-M. Chang, "Supportvector-machine-based method for automated steel bridge rust assessment," Automation in Construction, vol. 23, pp. 9-19, 2012.

[7] M. Silva, A. Santos, E. Figueiredo, R. Santos, C. Sales, and J. C. W. A. Costa, "A novel unsupervised approach based on a genetic algorithm for structural damage detection in bridges," Engineering Applications of Artificial Intelligence, vol. 52, pp. 168-180, 2016.

[8] W. T. Yeung and J. W. Smith, "Damage detection in bridges using neural networks for pattern recognition of vibration signatures," Engineering Structures, vol. 27, no. 5, pp. 685-698, 2005.

[9] M. Mehrjoo, N. Khaji, H. Moharrami, and A. Bahreininejad, "Damage detection of truss bridge joints using Artificial Neural Networks," Expert Systems with Applications, vol. 35, no. 3, pp. 1122-1131, 2008.

[10] S.-F. Jiang, C.-M. Zhang, and S. Zhang, "Two-stage structural damage detection using fuzzy neural networks and data fusion techniques," Expert Systems with Applications, vol. 38, no. 1, pp. 511-519, 2011.

[11] J. Min, S. Park, C. Yun, C. Lee, and C. Lee, "Impedance-based structural health monitoring incorporating neural network technique for identification of damage type and severity," Engineering Structures, vol. 39, pp. 210-220, 2012.

[12] J. Shu, Z. Zhang, I. Gonzalez, and R. Karoumi, "The application of a damage detection method using Artificial Neural Network and train-induced vibrations on a simplified railway bridge model," Engineering Structures, vol. 52, pp. 408-421, 2013.

[13] R. Ugalde, J.-C. Carmona, V. M. Alvarado, J. Reyes-Reyes, and J. Mantilla, "Computational cost improvement of neural network models in black box nonlinear system identification," Neurocomputing, vol. 166, pp. 96-108, 2015.

[14] O. Abdeljaber and O. Avci, "Nonparametric structural damage detection algorithm for ambient vibration response: Utilizing artificial neural networks and self-organizing maps," Journal of Architectural Engineering, vol. 22, no. 2, Article ID 04016004, 2016.

[15] Z. Zhang and A. E. Aktan, "Application of modal flexibility and its derivatives in structural identification," Research in Nondestructive Evaluation, vol. 10, no. 1, pp. 43-61, 1998.

[16] V. B. Dawari and G. R. Vesmawala, "Modal curvature and modal flexibility methods for honeycomb damage identification in reinforced concrete beams," in Proceedings of the 3rd Nirma University International Conference on Engineering, NUiCONE 2012, pp. 119-124, ind, December 2012.

[17] Y. Wang, M. Liang, and J. Xiang, "Damage detection method for wind turbine blades based on dynamics analysis and mode shape difference curvature information," Mechanical Systems and Signal Processing, vol. 48, no. 1-2, pp. 351-367, 2014.

[18] D. Wu and S. S. Law, "Damage localization in plate structures from uniform load surface curvature," Journal of Sound and Vibration, vol. 276, no. 1-2, pp. 227-244, 2004.

[19] T. Liu, A. Q. Li, C. Q. Miao, and Z. J. Li, "Study of structural damage identification methods based on data fusion," Engineering Mechanics, vol. 25, no. 1, pp. 16-21, 2008.

[20] R. Pasquier and I. F. C. Smith, "Robust system identification and model predictions in the presence of systematic uncertainty," Advanced Engineering Informatics, vol. 29, no. 4, pp. 1096-1109, 2015.

[21] Z. Pawlak, Rough Sets: Theoretical Aspects of Reasoning about Data, Springer, Dordrecht, Netherlands, 1991.
[22] O. Addin, S. M. Sapuan, E. Mahdi, and M. Othman, "A NaïveBayes classifier for damage detection in engineering materials," Materials and Corrosion, vol. 28, no. 8, pp. 2379-2386, 2007.

[23] M. Li, C. Shang, S. Feng, and J. Fan, "Quick attribute reduction in inconsistent decision tables," Information Sciences, vol. 254, pp. 155-180, 2014.

[24] K. Zheng, J. Hu, Z. Zhan, J. Ma, and J. Qi, "An enhancement for heuristic attribute reduction algorithm in rough set," Expert Systems with Applications, vol. 41, no. 15, pp. 6748-6754, 2014.

[25] Y. Robert-Nicoud, B. Raphael, O. Burdet, and I. F. C. Smith, "Model identification of bridges using measurement data," Computer-Aided Civil and Infrastructure Engineering, vol. 20, no. 2, pp. 118-131, 2005.

[26] S. Saitta, P. Kripakaran, B. Raphael, and I. F. C. Smith, "Improving system identification using clustering," Journal of Computing in Civil Engineering, vol. 22, no. 5, pp. 292-302, 2008.

[27] H. Cao and X. P. Lin, "Noise simulation in structural damage identification," Journal of Vibration and Shock, vol. 29, no. 5, pp. 106-109, 2010.

[28] W. R. Wickramasinghe, D. P. Thambiratnam, T. H. T. Chan, and T. Nguyen, "Vibration characteristics and damage detection in a suspension bridge," Journal of Sound and Vibration, vol. 375, pp. 254-274, 2016. 


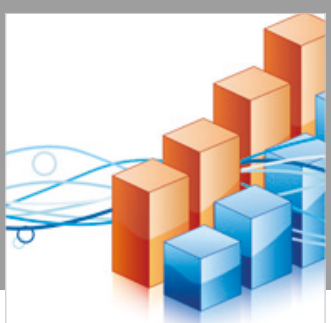

Advances in

Operations Research

\section{-n-m}
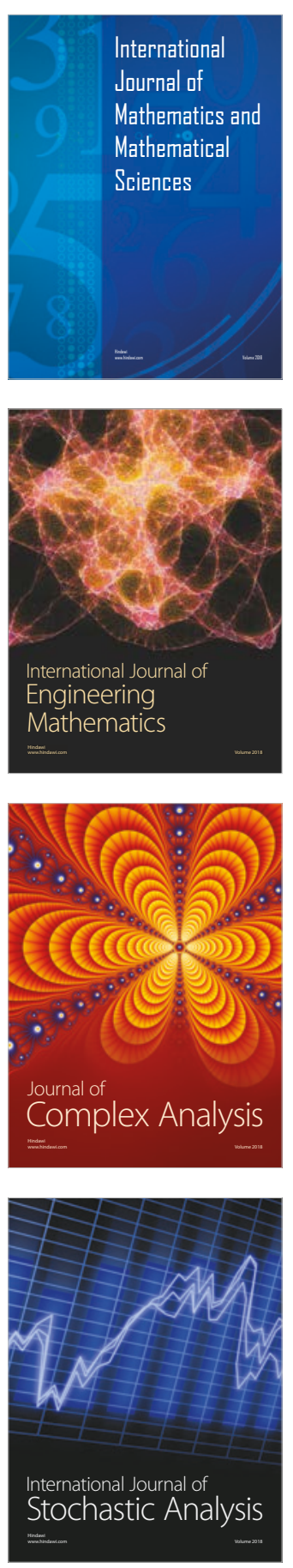
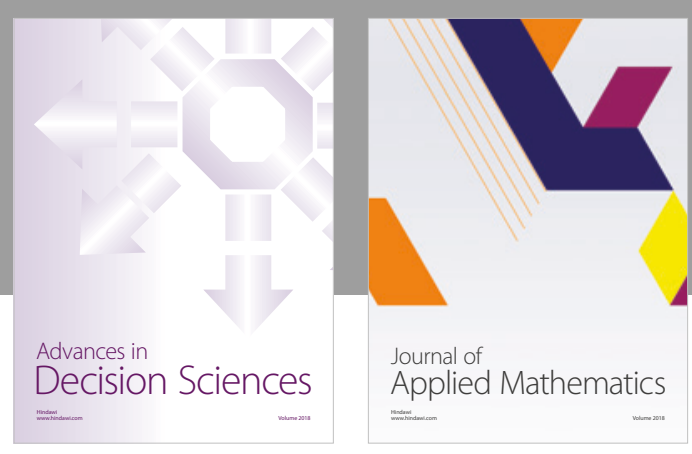

Journal of

Applied Mathematics
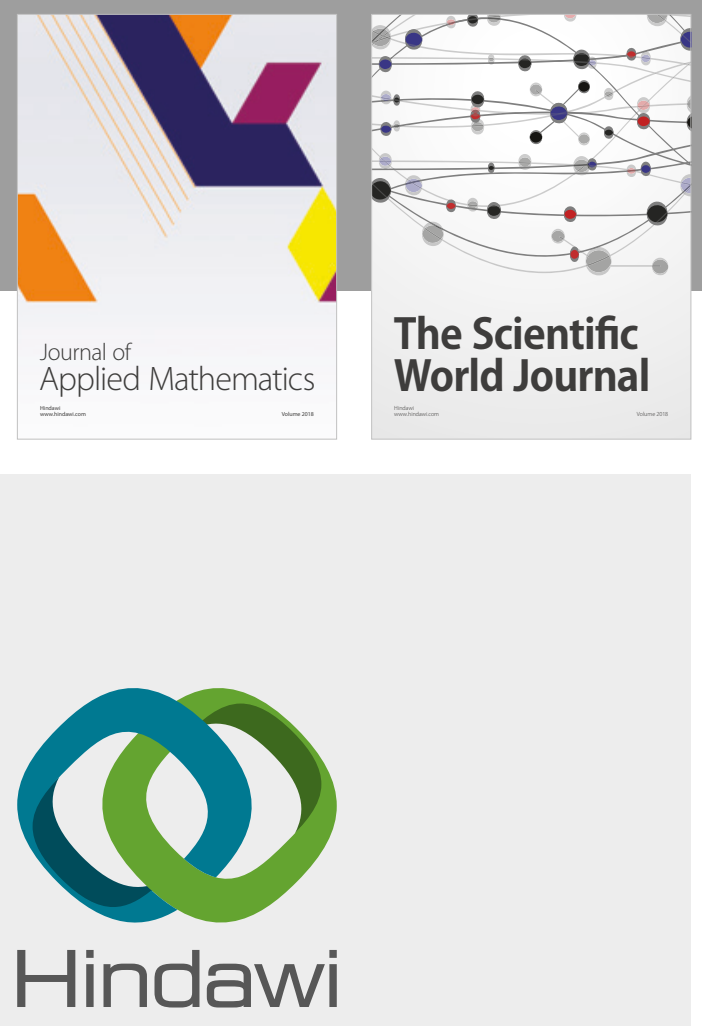

Submit your manuscripts at

www.hindawi.com

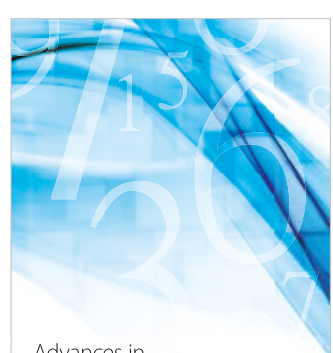

Advances in
Numerical Analysis
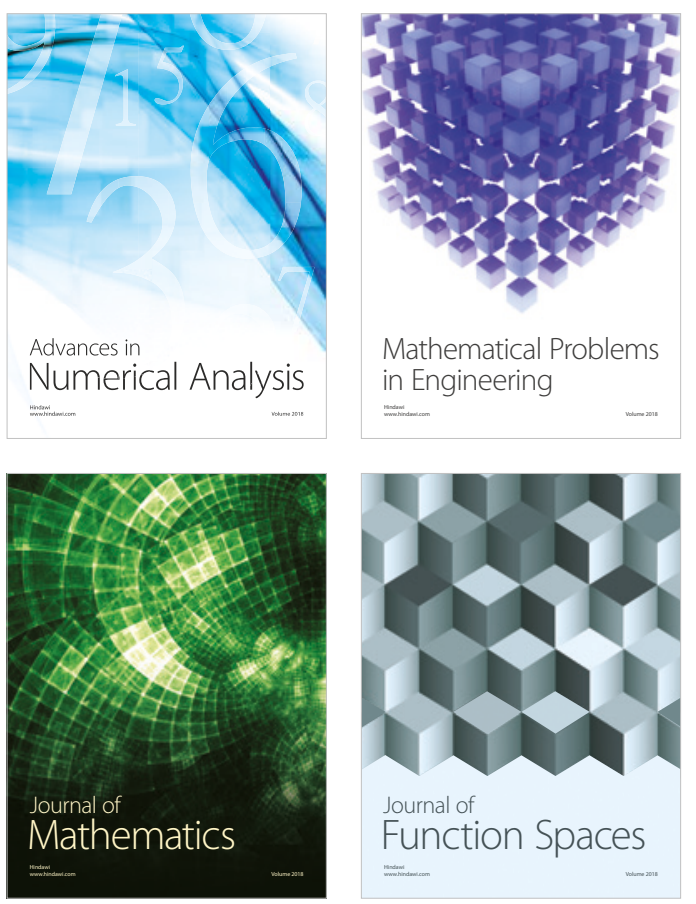

Mathematical Problems in Engineering

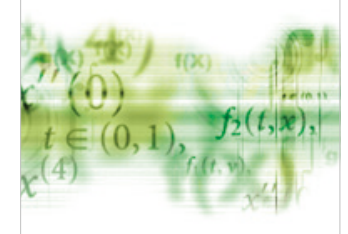

International Journal of

Differential Equations

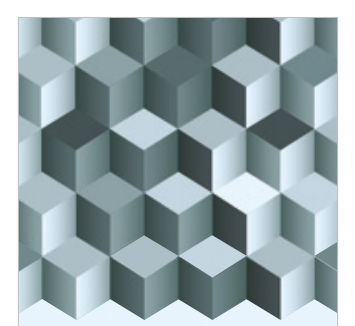

Journal of

Function Spaces
The Scientific

World Journal

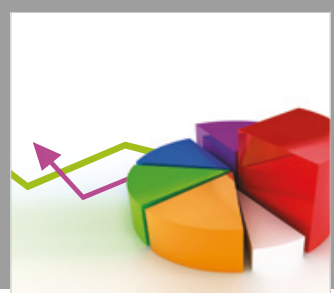

Journal of

Probability and Statistics
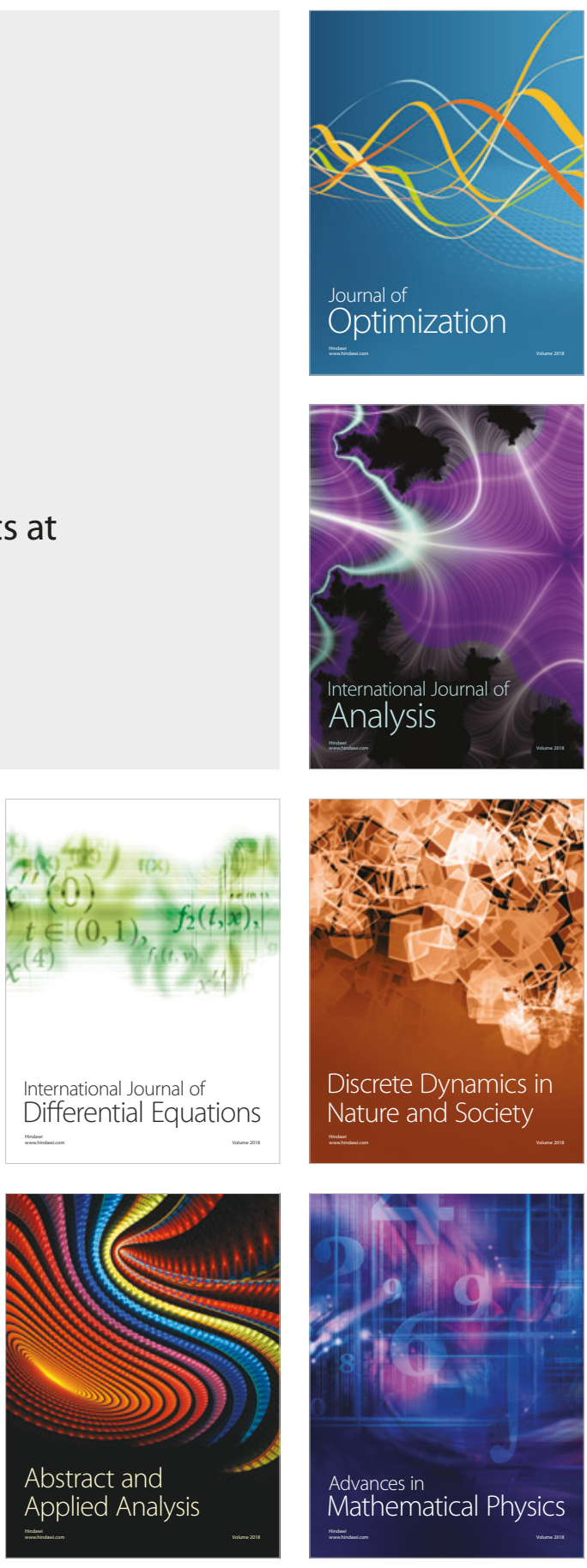